\title{
Endocarditis Due to Kytococcus schroeteri: Case Report and Review of the Literature
}

\author{
Basma Mnif, ${ }^{1}$ Inès Boujelbène, ${ }^{1}$ Fouzia Mahjoubi, ${ }^{1}$ Radouane Gdoura, ${ }^{1}$ Imen Trabelsi, ${ }^{3}$ \\ Sana Moalla, ${ }^{1}$ Imed Frikha, ${ }^{2}$ Samir Kammoun, ${ }^{3}$ and Adnane Hammami ${ }^{1 *}$ \\ Laboratoire de Microbiologie, CHU Habib Bourguiba, Sfax 3029, Tunisia ${ }^{1}$; Service de chirurgie cardio-vasculaire, \\ CHU Habib Bourguiba, Sfax 3029, Tunisia ${ }^{2}$; and Service de cardiologie, CHU Hedi Chaker, Sfax 3029, Tunisia ${ }^{3}$
}

Received 16 October 2005/Returned for modification 13 November 2005/Accepted 29 December 2005

\begin{abstract}
We report the third case of endocarditis caused by the newly described micrococcal species Kytococcus schroeteri. A 49-year-old woman was admitted to the hospital with suspected prosthetic valve endocarditis. Five blood cultures and prosthetic valve cultures grew the same type of organism, initially identified as Micrococcus sp. Assignment to the genus Kytococcus was suggested by the arginine dihydrolase activity and resistance to oxacillin. After sequencing of the $16 \mathrm{~S}$ rRNA genes, the isolate was recognized as $K$. schroeteri. The patient was treated first with vancomycin combined with gentamicin and later with pristinamycin and rifampin. Three cases of $K$. schroeteri endocarditis described within a short period of time might indicate a specific pathogenicity of this new species. The isolation of kytococci from normally sterile sites should not be overlooked.
\end{abstract}

\section{CASE REPORT}

A 49-year-old woman was admitted to the hospital due to persistent fever for 1 week. She had a history of rheumatic heart disease and had undergone mitral valve replacement 10 years earlier. On admission, the patient denied any recent history of dental work, intravenous drug abuse, or diagnostic procedures. Her temperature was $38.5^{\circ} \mathrm{C}$, her blood pressure $110 / 60 \mathrm{~mm} \mathrm{Hg}$, and her pulse 88 beats/min. A systolic murmur was heard on cardiac auscultation, and the lungs were clear by auscultation and percussion. The findings of the rest of her physical examination were unremarkable. Laboratory tests revealed the following values: hemoglobin, $120 \mathrm{~g} /$ liter; white blood cells, $8.4 \times 10^{9} /$ liter; erythrocyte sedimentation rate, 100 $\mathrm{mm} / \mathrm{h}$; and C-reactive protein, $125 \mathrm{mg} /$ liter. The liver function tests, renal function tests, and serum electrolyte levels were all normal.

A transesophageal echocardiogram disclosed prosthesis disinsertion, with two voluminous and mobile vegetations involving the mitral prosthesis (sizes, 12 by $13 \mathrm{~mm}$ and 12 by 12 $\mathrm{mm}$ ). Mitral valve replacement was recommended. Six sets of blood cultures were drawn. Therapy with cefotaxime and amikacin was instituted, and valve replacement surgery was performed. Five blood cultures and prosthetic valve cultures yielded gram-positive cocci. According to the antimicrobial susceptibilities of the isolates, the therapy was replaced with vancomycin-gentamicin and later with pristinamycin-vancomycin, and our patient responded well to this combination therapy. Six weeks after admission, the patient was discharged from the hospital in good condition and continued to take oral rifampin and pristinamycin for 3 weeks.

Microbiology. Blood cultures were processed with a BacT/ Alert system. In five of the six blood cultures drawn on admis-

\footnotetext{
* Corresponding author. Mailing address: Department of Microbiology, Habib Bourguiba Hospital, Elfardaous Street, Sfax 3029, Tunisia. Phone: 21674 456450. Fax: 21674 246217. E-mail: adnene.hammami @rns.tn.
}

sion, growth was detected after an incubation of 72 to $94 \mathrm{~h}$. Subcultures on blood agar yielded circular, convex, smooth, muddy yellow, and nonhemolytic colonies of heterogeneous size after $24 \mathrm{~h}$ of incubation. These colonies increased in size to about 1.5 to $2.5 \mathrm{~mm}$ in diameter after $48 \mathrm{~h}$. A Gram stain of these colonies showed spherical cells, predominantly occurring in pairs, in tetrads, and occasionally in clusters. The vegetation and the prosthetic valve cultures grew the same type of colonies. All isolates displayed identical biotype and antimicrobial susceptibility, and so they were considered as a single strain. The causative organism was initially identified as Micrococcus sp., based on the typical Gram stain morphology, resistance to nitrofurantoin, susceptibility to bacitracin, and inability to grow anaerobically. The reactions obtained by use of an ID32 STAPH gallery (bioMérieux) were similar to those of Micrococcus luteus or Micrococcus lylae. Nevertheless, this species differs from micrococci by resistance to penicillin and methicillin and by arginine dihydrolase activity. Besides, the colonies of this species grow more slowly than those of other micrococci. To obtain an accurate identification of this strain, molecular studies were conducted.

The 16S rDNA was amplified using universal 16S rRNA gene primers NW9, 5'-GCTAACTACGTGCCAGCAG-3', and NW17, 5'-TAAGGGCCATGATGACTTGAC-3'. The purified PCR products were sequenced with an ABI PRISM BigDye sequencing kit (v1.1) and analyzed with an ABI 3100-Avant genetic analyzer (Applied Biosystems) (4).

Upon comparison of the sequence generated in this assay with the publicly available bacterial sequences obtained from the National Center for Biotechnology Information (http://www.ncbi .nlm.nih.gov), the bacterium was identified as Kytococcus schroeteri.

Antimicrobial susceptibility tests were performed by the disk diffusion method (Bio-Rad disk) on Mueller-Hinton agar. The results were expressed as susceptible, intermediate, or resistant according to the criteria of CLSI (formerly NCCLS) (11). MICs of penicillin G, oxacillin, cefotaxime, vancomycin, and teicoplanin were determined by Etest (AB Biodisk) and inter- 
preted according to the criteria established for staphylococci in 2000 by CLSI (10).

By these tests, the strain demonstrated resistance to penicillin $\mathrm{G}$ (MIC, $>256 \mu \mathrm{g} / \mathrm{ml}$ ), oxacillin (MIC, $>256 \mu \mathrm{g} / \mathrm{ml}$ ), cefoxitin, cefotaxime (MIC, $>256 \mu \mathrm{g} / \mathrm{ml}$ ), kanamycin, tobramycin, erythromycin, and fusidic acid and susceptibility to imipenem (MIC, $0.50 \mu \mathrm{g} / \mathrm{ml}$ ), gentamicin, trimethoprim, tetracycline, vancomycin (MIC, $0.25 \mu \mathrm{g} / \mathrm{ml}$ ), teicoplanin (MIC, $0.25 \mu \mathrm{g} / \mathrm{ml}$ ), pristinamycin, and rifampin.

Discussion. The genus Kytococcus was established in 1995 with the type species Kytococcus sedentarius and was classified as a member of the family Dermatophilaceae (15). There are few reports documenting the role of Kytococcus species in human invasive diseases. This may be due in part to microbiologists' and clinicians' lack of familiarity with unusual micrococci, such as $K$. schroeteri, which leads to misidentification of these organisms as unspecified micrococci or misdiagnosis as environmental contaminants. In addition to difficulties with identification of clinical isolates, antibiotic susceptibility data for Kytococcus species are limited, making optimal treatment recommendations problematic. As microbiological and molecular techniques for their identification improve and awareness of their pathogenic potential increases, Kytococcus species are likely to emerge as increasingly important bacterial pathogens.

Micrococcal species are considered common commensals of the human skin. Their role as pathogens, when isolated from clinical specimens, can be difficult to determine. Since early reports of endocarditis caused by gram-positive cocci did not reliably differentiate between micrococci and coagulase-negative staphylococci, the frequency of micrococcal endocarditis is difficult to ascertain and might be underestimated. However, some cases of endocarditis attributed to M. lylae, M. luteus, $K$. sedentarius, and unspecified micrococci have been reported (14).

$K$. schroeteri is a new micrococcal species recently implicated in endocarditis. Cells are spherical and occur predominantly in pairs or tetrads. Colonies are as large as $2.5 \mathrm{~mm}$ in diameter (after $48 \mathrm{~h}$ of incubation), muddy yellow, nonhemolytic, circular, entire, convex, and smooth, and they develop rather slowly. This species is negative by oxidase test, positive for alkaline phosphatase, arginine dihydrolase, and pyrazinamidase, and negative for lecithinase, $\beta$-galactosidase, and urease. It hydrolyzes gelatin and Tween 80 but does not hydrolyze esculin or starch. The optimum growth temperature is $37^{\circ} \mathrm{C}$. K. schroeteri is resistant to penicillin $\mathrm{G}$, oxacillin, and erythromycin and susceptible to imipenem, chloramphenicol, ciprofloxacin, gentamicin, tetracycline, vancomycin, and teicoplanin. The type strain is strain Muenster $2000(1,15)$.

The first case of $K$. schroeteri endocarditis found in the literature, as a result of a Medline search, was reported by Becker et al. in 2003 (2). K. schroeteri was characterized and classified as a second species of the genus Kytococcus in 2002 (1). K. sedentarius, the type species of this genus, has been documented to be a causative organism in various infections $(6,12,13)$. The second case was described by Cécile Le Brun in 2005 (7). Here, we describe what is, to our knowledge, the third case of prosthetic valve endocarditis caused by the newly described micrococcal species $K$. schroeteri and we review the literature on $K$. schroeteri endocarditis.

The three patients had prior heart disease (a prosthetic valve which was involved in the infection). However, they did not have an evident predisposing event. In the first case (2), perioperative contamination was suggested, as K. schroeteri endocarditis had developed in the patient $<3$ months after she underwent cardiac surgery. Our patient and the patient from the second case, who were immunocompetent, had no recent history of intravenous drug administration or dental treatment. Endocarditis was probably caused by hematogenous spread in the two cases. As the natural habitat of $K$. schroeteri remains unknown, the mode of contamination also remains unclear. In fact, Le Brun et al. (7) were unable to recover $K$. schroeteri from the skin or mucous membranes of their patients.

The initial presentation of the endocarditis was nonspecific, including flu-like symptoms, fever, and dyspnea. The diagnosis was based on persistent $K$. schroeteri bacteremia and typical echocardiographic findings in the three cases, which fulfill the Duke criteria for clinically definite infective endocarditis (8). However, although the isolate of $K$. schroeteri has been identified, its clinical significance has always been underestimated; its recovery from the prosthetic material confirmed the implication of $K$. schroeteri in two cases of endocarditis.

In the two previously reported cases of $K$. schroeteri endocarditis, therapy with vancomycin, gentamicin, and rifampin was performed, resulting in bacteriologic eradication and clinical cure. Our patient was treated with vancomycin combined with gentamicin and later with pristinamycin and rifampin. As the optimal duration of antibiotic treatment of prosthetic valve endocarditis caused by uncommon pathogens is not known, we discontinued the therapy after 6 weeks.

Data on antimicrobial susceptibilities of micrococci are rare. In contrast to Micrococcus spp., for which Kytococcus spp. may easily be mistaken, Kytococcus spp. are usually penicillin and oxacillin resistant. The property of resistance to both benzylpenicillin and methicillin is considered specific for the genus Kytococcus, but the mechanism of this resistance has not been yet explored. The most promising antibiotic regimen suggested for the treatment of $K$. schroeteri is likely to be a combination of vancomycin, gentamicin, and rifampin. Overall, rifampin shows the highest activity against all micrococcal species (17). Furthermore, the addition of rifampin to many antibiotics, such as aminoglycoside, co-trimoxazole, or vancomycin, resulted in enhanced serum bactericidal activity for patients with prosthetic valve endocarditis due to methicillin-resistant Staphylococcus and Micrococcus species (3).

Micrococcus-like organisms are constituents of the normal human buccal flora (16). Despite their low virulence, these organisms may become pathogenic $(14,18)$, colonizing the surface of heart valves. In this report, the recovery of $K$. schroeteri from five blood cultures and prosthetic valve cultures implies the diagnosis of infective endocarditis. $K$. schroeteri is likely to emerge as an increasingly important bacterial pathogen, since four infections due to $K$. schroeteri were described within a short period of time (3 years): three cases of $K$. schroeteri endocarditis and recently a fatal $K$. schroeteri bacteremic pneumonia in a patient undergoing chronic corticosteroid therapy (9). Further investigations should be carried out to identify the virulence determinants of this species. 
This case demonstrates the usefulness of sequencing of the 16S rRNA genes for the identification of $K$. schroeteri, because the commercial systems are not able to identify this species. However, gram-positive cocci that are arranged in tetrads and that are oxacillin resistant and arginine dihydrolase positive should be recognized as potential Kytococcus species (15).

Our experience presented here reemphasizes the fact that $K$. schroeteri can be a true pathogen in some circumstances, especially in patients with an implanted prosthesis. When $K$. schroeteri is isolated from blood, amplification and sequencing of $16 \mathrm{~S}$ rRNA genes are promising tools for the identification of the pathogen.

We thank S. Ben Hamed, proficient in the English language, for checking the manuscript. We also thank S. Bouaziz for helpful assistance with sequencing of the $16 \mathrm{~S}$ rDNA.

\section{REFERENCES}

1. Becker, K., P. Schumann, J. Wüllenweber, M. Schulte, H. P. Weil, E. Stackebrandt, G. Peters, and C. von Eiff. 2002. Kytococcus schroeteri sp. nov., a novel gram-positive actinobacterium isolated from a human clinical source. Int. J. Syst. Evol. Microbiol. 52:1609-1614.

2. Becker, K., J. Wüllenweber, H. J. Odenthal, M. Moeller, P. Schumann, G. Peters, and C. von Eiff. 2003. Prosthetic valve endocarditis due to Kytococcus schroeteri. Emerg. Infect. Dis. 9:1493-1494.

3. Colebunders, R., J. P. Ursi, S. Pattyn, and J. Snoeck. 1985. Prosthetic valve endocarditis due to methicillin-resistant Staphylococcus epidermidis and Micrococcus species successfully treated with rifampicin combined with other antibiotics. J. Infect. 11:35-39.

4. Drancourt, M., C. Bollet, R. Carlioz, R. Martelin, J. P. Gayral, and D. Raoult. 2000. 16S ribosomal DNA sequence analysis of a large collection of the environmental and clinical unidentifiable bacterial isolates. J. Clin. Microbiol. 38:3623-3630.

5. Reference deleted.
6. Greene, G. R., C. M. Ninch, and E. L. Fohz. 1980. Cerebral cyst infection with Micrococcus sedentarius. J. Neurosurg. 52:126-128.

7. Le Brun, C., J. Bouet, P. Gautier, J. L. Avril, and O. Gaillot. 2005. Kytococcus schroeteri endocarditis. Emerg. Infect. Dis. 11:179-180.

8. Li, J. S., D. J. Sexton, N. Mick, R. Nettles, V. G. Fowler, Jr., T. Ryan, T. Bashore, and G. R. Corey. 2000. Proposed modifications to the Duke criteria for the diagnosis of infective endocarditis. Clin. Infect. Dis. 30:633-638.

9. Mohammedi, I., C. Berchiche, K. Becker, K. Belkhouja, D. Robert, C. von Eiff, and J. Etienne. 2005. Fatal Kytococcus schroeteri bacteremic pneumonia. J. Infect. 51:E11-E13.

10. National Committee for Clinical Laboratory Standards. 2000. Methods for dilution antimicrobial susceptibility tests for bacteria that grow aerobically. Approved standard M7-A4. National Committee for Clinical Laboratory Standards, Wayne, Pa.

11. National Committee for Clinical Laboratory Standards. 2000. Performance standards for antimicrobial disk susceptibility tests. Approved standard M2A7. National Committee for Clinical Laboratory Standards, Wayne, Pa.

12. Nordstrom, K. M., K. J. McGlinley, L. Cappiello, J. M. Zechman, and J. J. Leyden. 1987. Pitted keratolysis. The role of Micrococcus sedentarius. Arch. Dermatol. 123:1320-1325.

13. Old, D. C., and G. P. McNeill. 1979. Endocarditis due to Micrococcus sedentarius incertae sedis. J. Clin. Pathol. 32:951-952.

14. Seifert, H., M. Kaltheuner, and F. Perdreau-Remington. 1995. Micrococcus luteus endocarditis: case report and review of the literature. Zentbl. Bakteriol. 282:431-435.

15. Stackebrandt, E., C. Koch, O. Gvozdiak, and P. Schumann. 1995. Taxonomic dissection of the genus Micrococcus: Kocuria gen. nov., Nesterenkonia gen. nov., Kytococcus gen. nov., Dermacoccus gen. nov., and Micrococcus Cohn 1872 gen. emend. Int. J. Syst. Bacteriol. 45:682-692.

16. Szczerba, I., and Z. Krzeminski. 2002. Occurrence and number of bacteria from the Micrococcus, Kocuria, Nesterenkonia, Kytococcus and Dermacoccus genera on skin and mucous membranes in humans. Med. Dosw. Mikrobiol. 55:67-74.

17. von Eiff, C., M. Herrmann, and G. Peters. 1995. Antimicrobial susceptibilities of Stomatococcus mucilaginosus and of Micrococcus spp. Antimicrob. Agents Chemother. 39:268-270.

18. von Eiff, C., N. Kuhn, M. Herrmann, S. Weber, and G. Peters. 1996. Micrococcus luteus as a cause of recurrent bacteremia. Pediatr. Infect. Dis. J. 15:711-713. 